\title{
A NOTE TO A COMMON CAUSE IN QUANTUM LOGIC
}

\section{ABSTRACT}

We study the notion of a common cause in quantum logics as a type of causal relations (see [13]). We review maps like conditional state, s-map as basic tools of our constructions. Finally, we show some properties of such notions in quantum logics.
Address:

Department of Mathematics and Descriptive Geometry Faculty of Civil Engineering

\section{KEY WORDS}

- orthomodular lattice,

- s-map,

- conditional state,

- common cause.

\section{INTRODUCTION}

Probability measure theory is the basis of many constructions in quantum structures. It can be understood as a mathematical model for the intuitive notion of uncertainty [6]. Without probability measure theory all the stochastic models in physics, biology and economics either would not have been developed or would not be rigorous. The modern period of probability measure theory is connected to the names such as S.N. Bernstein (1880-1968), E. Borel (1871-1956) and A.N. Kolmogorov (1903-1987).

Moreover, in Physics, a probability space cannot solve the problem of incompatible elements which appears in the Von Neumann model (1932). For that reason, researchers, for example (Von Neumann) looked for new techniques that could explain the situation where the elements are either compatible or incompatible. In connection with classical concept of probability measure theory trying to solve problems of incompatible random events, many researchers such as $[1,10,11]$ have introduced equivalent approaches. One of these approaches is called the orthomodular lattice (OML). Many variant maps have been defined on an orthomodular lattice to solve different types of quantum structure problems.

One of the basic multivariate functions that has been proposed in quantum logic is a function called s-map, which was introduced by Olga Nánásiová in 2003. This function plays a main role in our constructions.

\section{BASIC NOTIONS}

In this part, we review basic definitions, properties and propositions that we will need to build our constructions. These concepts have been studied in detail $[1,3,12,17]$. We suppose that the triple $(\Omega, \mathrm{F}$, $P)$ is a probability space [6]. The elements of $\sigma$-algebra $\mathrm{F}$ are called random events. It is a well-known fact that $\forall A, B \in \mathrm{F}, A=(A \cap B) \cup$ 
$\left(A \cap B^{\mathrm{C}}\right)$. This property means that all random events are simultaneously measurable in a probability space. In this case, we say that $A$ and $B$ are compatible. Events $A, B \in \mathrm{F}$ are called independent, if $P=(A \cap B)=P(A) P(B)$. It is obvious that $A$ and $B$ are independent if and only if the conditional probability $P(B \mid A)=B$ ). Definition 1. 1 Let $(\Omega, \mathrm{F}, P)$ be a probability space. Then $C \in \mathrm{F}$ is a common cause to $A, B \in \mathrm{F}$ if the following conditions hold:

(1) $P(A \cap B \mid C)=P(A \mid C) P(B \mid C)$;

(2) $P\left(A \cap B \mid C^{c}\right)=P\left(A \mid C^{c}\right) P\left(B \mid C^{c}\right)$;

(3) $P(A \mid C)>P\left(A \mid C^{c}\right)$;

(4) $P(B \mid C)>P\left(B \mid C^{c}\right)$.

Now, we can introduce the definitions and basic properties of orthomodular lattice and state. These notions have been studied in detail $[1,12,17]$.

Definition 1.2 Let $L$ be a lattice with the greatest element $1_{\mathrm{L}}$, the smallest element $0_{\mathrm{L}}$ and partial ordering $\leq$, endowed with a unary operation $\perp: L \rightarrow L$, such that the following conditions hold:

(1) $\left(a^{\perp}\right)^{\perp}=a$;

(2) $a \leq b$ implies $b^{\perp} \leq a^{\perp}$;

(3) $a \vee a^{\perp}=1_{L}$;

(4) $a \leq b$ implies $b=a \vee\left(a^{\perp} \wedge b\right)$.

Then the system $\ell=\left(L, 0_{L}, 1_{L}, \vee, \wedge, \perp\right)$ is said to be an orthomodular lattice.

Condition (4) is called the orthomodular law. If the set $L$ is closed under countable lattice operations, then $\ell$ is called an orthomodular $\sigma$-lattice.

Let $a, b \in L$. We say that $a, b$ are orthogonal $(a \perp b)$, if $a \leq b^{\perp}$. We say that $a, b$ are compatible ( $a \leftrightarrow b$ ) if there exist mutually orthogonal elements $a_{0}, b_{0}, c \in L$ such that $a=a_{0} \vee c$ and $b=b_{0} \vee$ $c$. This means that $a \leftrightarrow b$ if and only if $a=(a \wedge b) \vee\left(a \wedge b^{\perp}\right)$ and $b=(a \wedge b) \vee\left(a^{\perp} \wedge b\right)$.

It is a well-known fact that $a=(a \wedge b) \vee\left(a \wedge b^{\perp}\right)$ for each $a, b \in L$. If, for each $a, b \in L, a \leftrightarrow b$, then $\ell$ is a Boolean algebra [11, 16].

Definition 1.3 Let $\ell$ be an orthomodular lattice. A state on $\ell$ is a map $\mathrm{m}: \mathrm{L} \rightarrow[0,1]$ with the following properties:

(1) $m\left(1_{L}\right)=1$

(2) $m\left(\vee_{i=1}^{n} a_{i}\right)=\sum_{i=1}^{n} m\left(a_{i}\right)$, for any $a_{1}, \ldots, a_{n} \in L$ such that $a_{i} \perp a_{j}$ whenever $i \neq j$.

Note that the state $m$ is a notion corresponding to the notion of probability measure, and it is clear that $m\left(0_{\mathrm{L}}\right)=0$. An orthomodular $\sigma$ - lattice L with a $\sigma$ - additive state $m$ is called a quantum logic $[12,17]$. It has been shown that there exist orthomodular lattices that admit no state $[11,2]$.

The notion of a conditional state in quantum logic was introduced by Nánásiová [7] and it is based on the definition of conditional probability in probability space, which was introduced in [16].
Definition 1. 4 Let $\ell$ be a quantum logic and $L_{0} \subset L-\left\{0_{L}\right\}$. A conditionalstate $\left(\sigma\right.$-conditional state) is a function $f: L \times L_{0} \rightarrow[0,1]$ that satisfies the following conditions:

(1) for each $a \in L_{0}, f(. \mid a)$ is a state on $L$;

(2) for each $a \in L_{0}, f(a \mid a)=1$;

(3) if $a_{\mathrm{n}} \in L_{0}$, where $n \in J \subset N$ and $a_{i} \perp a_{j}$ for $i, j \in J, i \neq j$ and $\vee_{n \in J} a_{n} \in L_{0}$ then for each $\mathrm{b} \in L f\left(b \mid \vee_{j \in J} a_{n}\right)=\sum_{n \in J} f\left(a_{n} \mid \vee_{j \in J} a_{n}\right) f\left(b \mid a_{n}\right)$.

Remark 1. 1 Let $(\Omega, \mathrm{F}, P)$ be a probability space. Then for all $A, B$ $\in \mathrm{F}$ such that, $P(A)>0, P(B)>0$, the following Bayesian property is true. $P(A) P(B \mid A)=P(B) P(A \mid B)$, but this in general is not true in quantum logic.

In the following, we introduce the definition of multivariate maps in quantum logic and their basic properties [7]. We start with a definition of an $\mathrm{n}$-dimensional s-map:

Definition 1.5 Let $\ell$ be a quantum logic. A function $p: L^{n} \rightarrow[0,1]$ is called an n-dimensional s-map if the following conditions hold:

1. $p(I, \ldots, I)=1$

2. If there exists $i$, such that $a_{i} \perp a_{i+1}$, then $p\left(a_{1}, \ldots a_{i}, a_{i+1}, \ldots, a_{n}\right)=0$;

3. If $a_{i} \perp b_{i}$, then $p\left(a_{1}, \ldots, a_{i} \vee b_{i}, \ldots, a_{n}\right)=p\left(a_{1}, \ldots, a_{i}, \ldots, a_{n}\right)+p\left(a_{1}, \ldots, b_{i}, \ldots, a_{n}\right)$.

In particular, for $n=2$, we can see that the conditions of the definition of an n-dimensional s-map are the following:

- $p\left(1_{L}, 1_{L}\right)=1$;

- If $a \perp b$, then $p(a, b)=0$;

- If $a \perp b$, then for any $c \in L$, $p(a \vee b, c)=p(a, c)+p(b, c)$; $p(c, a \vee b)=p(c, a)+p(c, b)$.

Let $W_{\mathrm{n}}$ be the set of all $\mathrm{n}$-dimensional s-maps $(n=1,2, \ldots)$. It is clear that for $p \in W_{1}$ is a state on $L$. In fact, an s-map $p$ is a notion corresponding to the measure of an intersection. Let $L$ be a quantum logic, $p \in W_{2}$. If $a \leftrightarrow b$, then $p(a, b)=p(b, a)$. Moreover, if $a \leftrightarrow$ $b$, then $p(a, b)=p(a \wedge b, a \wedge b)$. We say that $a$ and $b$ have a causal relation if $p(a, b) \neq p(b, a)$.

\section{INDEPENDENCE IN QUANTUM LOGIC}

The theory of independence of random variables is one of the main concepts of a probability space. It is very important, for example, in the definition of conditional probability, Bayesian relations, etc. Also, the independence of elements in quantum logic is as important as in a probability space and is more general because it can be used to describe a type of causal relations. We will focus on an approach concerning the independence of elements in quantum logic using an s-map (see [8]).

Definition 2. 1 Let $\ell$ be a quantum logic and $f$ be a conditional state. 
Let $b \in L, a, c \in L_{0}$ such that $f(c \mid a)=1$. Then $a, b$ are independent with respect to the state $f(. \mid c)$ with notation $\left(b \sim_{f} a\right) \quad[c]$ if and only if $f(b \mid c)=f(b \mid a)$.

Proposition 2. 1 Let $\ell$ be a quantum logic, $L_{0}=L-\{O\}$ and let $f$ be a conditional state. Then there exists an s-map $p_{f}: L^{2} \rightarrow[0,1]$ that satisfies.

$$
p_{f}(a, b)=f(a, b) f(a, I) .
$$

Proposition 2.2 Let $\ell$ be a quantum logic, $p \in W_{2}$ Let $L_{0}=\{b \in L ; p(b, b)>0\}$. Then

$$
f_{p}(a \mid b)=\frac{p(a, b)}{p(b, b)} \text { is a conditional state. }
$$

\section{COMMON CAUSES IN QUANTUM LOGICS}

In 1956 Reichenbach based the concept of common cause and in 1997 Redie introduced a definition of common cause in probability space as a type of causal relations.

\section{1 Interpretation of positively correlated elements in quantum logic}

In this part, we review the classical definition of positively correlated events in a probability space (see[13, 14]). We interpret such a notion in quantum logic.

Now let us denote $R(a, b)=p(a, b)-p(a, a) p(b, b) \forall a, b \in L$, where $p \in W_{2}$.

Definition 3. 1. 1 Let $\ell$ be a quantum logic and let $p \in W_{2}$. Elements $a, b \in L$ are called positively correlated if $R(a, b)=p(a, b)-p(a, a) p(b, b)>0$.

If $R(a, b)<0$, then $a, b$ are called negatively correlated.

Lemma 3. 1. 1 Let $\ell$ be a quantum logic, $p \in W_{2}$. Then the following relations are true

$$
R(a, b)=R\left(a^{\perp}, b^{\perp}\right)=-R\left(a, b^{\perp}\right)=-R\left(a^{\perp}, b\right), \quad \forall a, b \in L .
$$

Proof. Let $a, b \in L$ and $p \in W_{2}$. Then $p\left(a, b^{\perp}\right)=p(a, a)-p(a, b)$. Hence

$$
\begin{aligned}
R\left(a, b^{\perp}\right) & =p(a, a)-p(a, b)-p(a, a) p\left(b^{\perp}, b^{\perp}\right)= \\
& =p(a, a)\left(1-p\left(b^{\perp}, b^{\perp}\right)\right)-p(a, b)
\end{aligned}
$$

$R\left(a, b^{\perp}\right)=p(a, a) p(b, b)-p(a, b)=-R(a, b) .($ E.Q.D. $)$

Lemma 3.1.2 Let $\ell$ be a quantum logic, $p \in W_{2}$ Let $a, b \in L$. Then $R(a, b)$ has the following properties:

(1) $a \sim_{p} b \quad\left[1_{L}\right]$ if and only if $R(a, b)=0$
(2) $R(a, a)=p(a, a) p\left(a^{\perp}, a^{\perp}\right)$;

(3) $R\left(a, a^{\perp}\right)=-R(a, a)$;

(4) $R(a, b) \in\left[\frac{-1}{4}, \frac{1}{4}\right]$ for $a, b \in L$

(5) $R\left(1_{L}, 1_{L}\right)=R\left(0_{L}, 0_{L}\right)=R\left(1_{L}, 0_{L}\right)=R\left(0_{L}, 1_{L}\right)=0$;

(6) If $a \perp b$, then $R(a, b) \leq 0$;

(7) If $a \leftrightarrow b$, then $R(a, b)=R(b, a)$.

Proof. It is enough to show the proof of property number (4) because proofs of the other properties are straightforwardy. We firstly show that $R(a, b) \leq \frac{1}{4}$.

Let $a, b \in L$ and $\min \{p(a, a), p(b, b)\}=p(a, a)$. Then $R(a, b)=p(a, b)-p(a, a) p(b, b) \leq p(a, a)-p(a, a) p(b, b)$. Hence $R(a, b) \leq p(a, a) p\left(b^{\perp}, b^{\perp}\right)$. But $p(a, a) \leq p(b, b)$, thus $p\left(b^{\perp}, b^{\perp}\right) \leq p\left(a^{\perp}, a^{\perp}\right)$. Hence,

$R(a, b) \leq p(a, a) p\left(b^{\perp}, b^{\perp}\right) \leq p(a, a) p\left(a^{\perp}, a^{\perp}\right)=p(a, a)(1-p(a, a))$. Let us denote $p(a, a)=k$ Then for the real function $g(k)=k(1-\mathrm{k})$, it is a well-known fact that $\max (g(k)) \leq \frac{1}{4}$. Therefore, $R(a, b) t \leq \frac{1}{4}$. In the second part of the proof, we want to show that $R(a, b) \geq \frac{-1}{4}$. Let $a_{0}, b_{0} \in L$ such that $R\left(a_{0}, b_{0}\right)<\frac{-1}{4}$. Then $-R\left(a_{0}, b_{0}^{\perp}\right)<\frac{-1}{4}$. Hence, $R\left(a_{o}, b_{o}^{\perp}\right)>\frac{1}{4}$. This means that $R\left(a_{o}, b_{o}\right)>\frac{1}{4}$, which contradicts the first part of the proof. (Q.E.D.)

Proposition 3.1. 1 Let $\ell$ be a quantum logic, $p \in W_{2}$ and let $a, b$ $\in$ L. Then $-R(a, a) \leq R(a, b) \leq R(a, a)$

Proof. We start by showing that $R(a, b) \leq R(a, a)$. Let $a, b \in L$ such that $R(a, b)>R(a, a)$. Then $p(a, b)-p(a, a) p(b, b)>p(a, a)-p(a, a) p(a, a=$ $=p(a, b)+p\left(a, b^{\perp}\right)-p(a, a) p(a, a)$. Thus - $p(a, a) p(b, b)>p\left(a, b^{\perp}\right)-p(a, a) p(a, a)$; $p(a, a)[p(a, a)-p(b, b)]>p\left(a, b^{\perp}\right)$; $p(a, a)\left[p(a, a)-p(a, b)-p\left(a^{\perp}, b\right)\right]>p\left(a, b^{\perp}\right) ;$ $p(a, a) p\left(a, b^{\perp}\right)-p(a, a) p\left(a^{\perp}, b\right)>p\left(a, b^{\perp}\right)$; $p(a, a) p\left(a, b^{\perp}\right)-p\left(a, b^{\perp}\right)>p(a, a) p\left(a^{\perp}, b\right)$; $-p\left(a, b^{\perp}\right) p\left(a^{\perp}, a^{\perp}\right)>p(a, a) p\left(a^{\perp}, b\right) \geq 0$.

Thus $-p\left(a, b^{\perp}\right) p\left(a^{\perp}, a^{\perp}\right)>0$. And this contradicts the assumption, that $p \in W_{2}$. Therefore, $R(a, b) \leq R(a, a)$.

To prove that $-R(a, a) \leq R(a, b)$, the same technique of the proof above can be used. (Q.E.D.) 


\section{IDYHUSOOUSSLAL \\ of

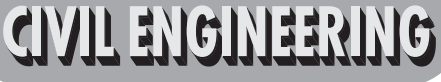

2010/4 PAGES $24-29$

Remark 3. 1. 1 Let $\ell$ be a quantum logic. We can see that $R\left(1_{L}, b\right)=R\left(0_{L}, b\right)=0$ for each $b \in L$.

Proposition 3. 1. 2 Let $L$ be a quantum logic, $p \in W_{2}$. If $a, b \in L$ such that $a \perp b$, then for each $c \in L$, it holds:

$R(a \vee b, c)=R(a, c)+R(b, c)$

$R(c, a \vee b)=R(c, a)+R(c, b)$.

Proof. We prove one of these two properties. Let $c \in L, a \perp b$ Then $R(a \vee b, c)=p(a \vee b, c)-p(a \vee b, a \vee b) p(c, c)$

$=p(a, c)+p(b, c)-[p(a, a)+p(b, b)] p(c, c)$

$=R(a, c)+R(b, c)$.

which means that $R$ is additive. (Q.E.D.)

\section{2 Definition of a Common Cause in Quantum Logic}

In 1997, Redei proposed a definition of common cause, which provides that events are compatible in a probability space. Similarly, we propose such a definition in quantum logic. One of the benefits of using the definition of a common cause in quantum logic is that the elements do not need to be compatible. This gives us more general constructions than constructions in a probability space. To introduce a proper definition of a common cause in quantum logic, we need to introduce concepts that help us build a definition of a common cause.

Definition 3.2. 1 Let $\ell$ be a quantum logic, $p \in W_{k+n}$ and $a_{1}, \ldots, a_{k}$, $b_{1}, \ldots, b_{n} \in W$. Aconditional s-map is a map $f_{k+n} \in W_{k}$ with the following property

$f_{k, n}\left(a_{1}, \ldots, a_{k} \mid b_{1}, \ldots, b_{n}\right)=\frac{p\left(a_{1}, \ldots, a_{k}, b_{1}, \ldots, b_{n}\right)}{p\left(1_{L}, \ldots, 1_{L}, b_{1}, \ldots, b_{n}\right)}$,

where $p\left(1_{L}, \ldots 1_{L}, b_{1}, \ldots, b_{n}\right)>0$.

Lemma 3. 2. 1 Let $\ell$ be a quantum logic, $p \in W_{3}$. Then 1. If $c \in L, p(c, c, c)>0$, and $f_{2,1}(., . \mid c)=\frac{p(., ., c)}{p(c, c, c)}$, then $f_{2,1}$ is an
s-map.

2. If $b, c \in L, p\left(1_{L}, b, c\right)>0$, and $f_{1,2}(. \mid b, c)=\frac{p(., b, c)}{p\left(1_{L}, b, c\right)}$, then $f_{1,2}$ is a state.

Proof. We only show (i) because (ii) follows directly from the first property (i).

(i) It is clear that $f_{2,1}\left(1_{L}, 1_{L} \mid c\right)=1$. Let $a, b \in L$ and let $a \perp b$. It is clear, that $f_{2,1}(a, b \mid c)=0$. We show only aditivity:

$f_{2,1}(a \vee b, d \mid c)=\frac{p(a \vee b, d, c)}{p(c, c, c)}=\frac{p(a, d, c)}{p(c, c, c)}+\frac{p(b, d, c)}{p(c, c, c)}=$

$=f_{2,1}(a, d \mid c)+f_{2,1}(b, d \mid c)$.

Similarly $f_{2,1}(d, a \vee b \mid c)=f_{2,1}(d, a \mid c)+f_{2,1}(d, b \mid c)$.

Therefore $f_{2,1}(., . \mid c)$ is an s-map. (Q.E.D.)
Lemma 3. 2. 2 Let $L$ be a quantum logic, $p \in W_{3}$ and let $a, b, c, d \in$ $L$ such that $b \perp c$, and $p\left(1_{L}, b, d\right)>0, p\left(1_{L}, c, d\right)>0$. Then $f_{1,2}(a \mid b \vee c, d)=f_{1,2}(b \mid b \vee c, d) f_{1,2}(a \mid b, d)+$

$+f_{1,2}(c \mid b \vee c, d) f_{1,2}(a \mid c, d)$

$f_{1,2}(a \mid d, b \vee c)=f_{1,2}(b \mid d, b \vee c) f_{1,2}(a \mid d, b)+$

$+f_{1,2}(c \mid d, b \vee c) f_{1,2}(a \mid d, c)$.

Proof. Let $b \perp c$ and $p\left(1_{L}, b \vee c, d\right)>0, p\left(1_{L}, b, d\right)>0, p\left(1_{L}, c, d\right)>0$. Then

$$
\begin{aligned}
f_{1,2}(a \mid b \vee c, d) & =\frac{p(a, b \vee c, d)}{p\left(1_{L}, b \vee c, d\right)}=\frac{p(a, b, d)}{p\left(1_{L}, b \vee c, d\right)}+\frac{p(a, c, d)}{p\left(1_{L}, b \vee c, d\right)} \\
& =\frac{p(a, b, d)}{p\left(1_{L}, b, d\right)} \frac{p\left(1_{L}, b, d\right)}{p\left(1_{L}, b \vee c, d\right)}+\frac{p(a, c, d)}{p\left(1_{L}, c, d\right)} \frac{p\left(1_{L}, c, d\right)}{p\left(1_{L}, b \vee c, d\right)}
\end{aligned}
$$

But $p\left(1_{L}, b, d\right)=p(b, b, d)=p(b, b \vee c, d)$,

$p\left(1_{L}, c, d\right)=p(c, c, d)=p(c, b \vee c, d)$. Therefore,

$$
\begin{aligned}
f_{1,2}(a \mid b \vee c, d) & =\frac{p(a, b, d)}{p\left(1_{L}, b, d\right)} \frac{p(b, b \vee c, d)}{p\left(1_{L}, b \vee c, d\right)}+\frac{p(a, c, d)}{p\left(1_{L}, c, d\right)} \frac{p(c, b \vee c, d)}{p\left(1_{L}, b \vee c, d\right)} \\
& =f_{1,2}(a \mid b, d) f_{1,2}(b \mid b \vee c, d)+f_{1,2}(a \mid c, d) f_{1,2}(c \mid b \vee c, d) .
\end{aligned}
$$

Similarly, we obtain that

$f_{1,2}(a \mid d, b \vee c)=f_{1,2}(b \mid d, b \vee c) f_{1,2}(a \mid d, b)+$

$$
+f_{1,2}(c \mid d, b \vee c) f_{1,2}(a \mid d, c) \text {. }
$$

(Q.E.D.)

\section{3 Independence of elements in quantum logic}

We turn now to the notion of the independence of elements with respect to an s-map in quantum logic. The definition can be proposed in the following way.

Definition 3.3.1 Let $\ell$ be a quantum logic, $p \in W_{k+n+t}$ and $a_{1}, \ldots, a_{k}$, $c_{1}, \ldots, c_{t} \in L$. If there exist $b_{1}, \ldots, b_{n}, \in L$ such that

$p\left(1_{L}, \ldots, 1_{L}, b_{1}, \ldots, b_{n}, c_{1}, \ldots, c_{t}\right)>0$ and

$f_{k, n+t}\left(a_{1}, \ldots, a_{k} \mid b_{1}, \ldots, b_{n}, c_{1}, \ldots, c_{t}\right)=$

$=f_{k, n+t}\left(a_{1}, \ldots, a_{k} \mid 1_{L}, \ldots, 1_{L}, c_{1}, \ldots, c_{t}\right)$,

then we say that $\left(a_{1}, \ldots, a_{k}\right)$ is independent to $\left(b_{1}, \ldots, b_{n}\right)$ with respect to $\left(c_{1}, \ldots, c_{t}\right)$ (denoted by $\left(a_{1}, \ldots, a_{k}\right) \sim_{f_{k, n+t}}\left(b_{1}, \ldots, b_{n}\right)\left[c_{1}, \ldots, c_{t}\right]$ ).

Lemma 3. 3. 1 Let $L$ be a quantum $\operatorname{logic} p \in W_{3}$, and $a, b, c, \in L$ If $a \sim_{f_{1,2}} b[c]$,

then $f_{2,1}(a, b \mid c)=f_{2,1}(a, a \mid c) f_{2,1}(b, b \mid c)$.

Proof. Let $a, b, c, \in L$ such that for $a \sim f_{f_{1}}, b[c]$. Then

$f_{2,1}(a, b \mid c)=\frac{p(a, b, c)}{p\left(1_{L}, 1_{L}, c\right)}=\frac{p(a, b, c)}{p\left(1_{L}, b, c\right)} \frac{p\left(1_{L}, b, c\right)}{p\left(1_{L}, 1_{L}, c\right)}=f_{1,2}(a \mid b, c) f_{1,2}\left(b \mid 1_{L}, c\right)$

Because $f_{1,2}(a \mid b, c)=f_{1,2}\left(a \mid 1_{L}, c\right)$, 


\section{YALSOUDSILL \\ 0)

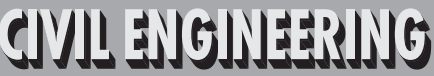

2010/4 PAGES $24-29$

then

$f_{2,1}(a, b \mid c)=f_{1,2}\left(a \mid 1_{L}, c\right) f_{1,2}\left(b \mid 1_{L}, c\right)$.

Hence

$f_{1,2}\left(a \mid 1_{L}, c\right)=\frac{p\left(a, 1_{L}, c\right)}{p\left(1_{L}, 1_{L}, c\right)}=\frac{p(a, a, c)}{p\left(1_{L}, 1_{L}, c\right)}=f_{2,1}(a, a \mid c)$,

then $f_{2,1}(a, b \mid c)=f_{2,1}(a, a \mid c) f_{2,1}(b, b \mid c)$ (Q.E.D.)

In particular, let $f_{2,1}$ be a conditional s-map and $f_{1,2}$ be a conditional state. We can see that if for $a, b \in L$ there exists $c \in L$ such that $a \sim{ }_{f_{21}} b[c]$, then

$f_{2,1}(a, b \mid c)=f_{1,2}(a \mid b, c) f_{1,2}\left(b \mid 1_{L}, c\right)$

Since we have prepared the background that a common cause is based upon, we are now able to put a proper definition of a common cause in quantum logic.

Definition 3. 3. 2 Let $\ell$ be a quantum logic, $f_{2,1}$ be a conditional s-map and let $L_{0}=\left\{d \in L: f_{21}\left(d, d \mid 1_{L}\right)>0\right\}$. A common cause of $(a, b) \in L^{2}$ is an element $c \in L_{0}$, such that $c^{\perp} \in L_{0}$ and satisfies the following conditions:

(1) $f_{2,1}(a, b \mid c)=f_{2,1}(a, a \mid c) f_{2,1}(b, b \mid c)$

(2) $f_{2,1}\left(a, b \mid c^{\perp}\right)=f_{2,1}\left(a, a \mid c^{\perp}\right) f_{2,1}\left(b, b \mid c^{\perp}\right)$

(3) $f_{2,1}(a, a \mid c)>f_{2,1}\left(a, a \mid c^{\perp}\right)$

(4) $f_{2,1}(b, b \mid c)>f_{2,1}\left(b, b \mid c^{\perp}\right)$

Proposition 3. 3. 1 Let $\ell$ be a quantum logic, $p \in W_{3}$ and $f_{2,1}$ be a conditional s-map generated by $p$. If $c \in L_{0}$ is a common cause of $a, b \in L$, then $R(a, b)>0$.

Proof. We show that

$R(a, b)=p\left(a, b, 1_{L}\right)-p(a, a, a) p(b, b, b)$

$$
\begin{aligned}
= & p\left(1_{L}, 1_{L}, c\right) p\left(1_{L}, 1_{L}, c^{\perp}\right)\left[f_{2,1}(a, a \mid c)-f_{2,1}\left(a, a c^{\perp}\right)\right] \\
& {\left[f_{2,1}(b, b c)-f_{2,1}\left(b, b c^{\perp}\right)\right]>0 . }
\end{aligned}
$$

Let $c \in L, p(c, c, c)>0$ be a common cause of $a, b \in L$. It is clear that $p(a, a, a)=p\left(1_{L}, a, a\right)=p\left(1_{L}, 1_{L}, a\right)$.

Let us denote $\forall u \in L^{3} p_{3}(u)=p(u, u, u)$ and $f_{2,1}(u \mid v)=\frac{p(u, u, v)}{p(v, v, v)}$, where $v \in L_{0}$. Then

$$
\begin{aligned}
R(a, b)=p\left(a, b, 1_{L}\right)-p_{3}(a) p_{3}(b) & =p\left(a, b, c \vee c^{\perp}\right)-p_{3}(a) p_{3}(b) \\
& =p(a, b, c)+p\left(a, b, c^{\perp}\right)-p_{3}(a) p_{3}(b) .
\end{aligned}
$$

Let $p_{3}(c)>0$. Thus, the terms $p(a, b, c), p\left(a, b, c^{\perp}\right), p_{3}(a), p_{3}(b)$ can be written in the following form

$p(a, a, c)+p\left(a, a, c^{\perp}\right)=f_{2,1}(a \mid c) p_{3}(c)+f_{2,1}\left(a \mid c^{\perp}\right) p_{3}\left(c^{\perp}\right)$

$p(b, b, c)+p\left(b, b, c^{\perp}\right)=f_{2,1}(b \mid c) p_{3}(c)+f_{2,1}\left(b \mid c^{\perp}\right) p_{3}\left(c^{\perp}\right)$.
We obtain that

$f_{2,1}(a, b \mid c) p_{3}(c)=f_{2,1}(a \mid c) f_{2,1}(b \mid c) p_{3}(c)$

$f_{2,1}\left(a, b \mid c^{\perp}\right) p_{3}\left(c^{\perp}\right)=f_{2,1}\left(a \mid c^{\perp}\right) f_{2,1}\left(b \mid c^{\perp}\right) p_{3}\left(c^{\perp}\right)$

Now, substitute equations (1), (2) in $p(a, b, c), p\left(a, b, c^{\perp}\right)$, we obtain

$p\left(a, b, 1_{L}\right)=f_{2,1}(a \mid c) f_{2,1}(b \mid c) p_{3}(c)+f_{2,1}\left(a \mid c^{\perp}\right) f_{2,1}\left(b \mid c^{\perp}\right) p_{3}\left(c^{\perp}\right)$.

Hence

$$
\begin{aligned}
p_{3}(a) p_{3}(b)= & {\left[f_{2,1}(a \mid c) p_{3}(c)+f_{2,1}\left(a \mid c^{\perp}\right) p_{3}\left(c^{\perp}\right)\right] } \\
& {\left[f_{2,1}(b \mid c) p_{3}(c)+f_{2,1}\left(b \mid c^{\perp}\right) p_{3}\left(c^{\perp}\right)\right] } \\
= & f_{2,1}(a \mid c) f_{2,1}(b \mid c) p_{3}^{2}(c)+f_{2,1}\left(a \mid c^{\perp}\right) f_{2,1}\left(b \mid c^{\perp}\right) p_{3}^{2}\left(c^{\perp}\right) \\
+ & f_{2,1}(a \mid c) f_{2,1}\left(b \mid c^{\perp}\right) p_{3}(c) p_{3}\left(c^{\perp}\right)+ \\
+ & f_{2,1}\left(a \mid c^{\perp}\right) f_{2,1}(b \mid c) p_{3}(c) p_{3}\left(c^{\perp}\right) .
\end{aligned}
$$

Further, subtract $p_{3}(a) p_{3}(b)$ from $p(a, b, I)$ and rearrange the terms of the quality above, we obtain

$R(a, b)=p_{3}\left(a, b, 1_{L}\right)-p_{3}(a) p_{3}(b)=$

$$
\begin{aligned}
& =f_{2,1}(a \mid c) f_{2,1}(b \mid c)\left[p_{3}(c)-p_{3}^{2}(c)\right] \\
& +f_{2,1}\left(a \mid c^{\perp}\right) f_{2,1}\left(b \mid c^{\perp}\right)\left[p_{3}\left(c^{\perp}\right)-p_{3}^{2}\left(c^{\perp}\right)\right] \\
& -p_{3}(c) p_{3}\left(c^{\perp}\right)\left[f_{2,1}(a \mid c) f_{2,1}\left(b \mid c^{\perp}\right)+f_{2,1}\left(a \mid c^{\perp}\right) f_{2,1}(b \mid c)\right]
\end{aligned}
$$

It is clear that $p_{3}(c)-p_{3}^{2}(c)=p_{3}(c) p_{3}\left(c^{\perp}\right)$, and $p_{3}\left(c^{\perp}\right)-p_{3}^{2}\left(c^{\perp}\right)=p_{3}(c) p_{3}\left(c^{\perp}\right)$, thus

$R(a, b)=p\left(a, b, 1_{L}\right)-p_{3}(a) p_{3}(b)=$

$$
\begin{aligned}
=p_{3}(c) p_{3}\left(c^{\perp}\right) & {\left[f_{2,1}(b \mid c)\left(f_{2,1}(a \mid c)-f_{2,1}\left(a \mid c^{\perp}\right)\right)\right.} \\
- & \left.f_{2,1}\left(b \mid c^{\perp}\right)\left(f_{2,1}(a \mid c)-f_{2,1}\left(a \mid c^{\perp}\right)\right)\right]
\end{aligned}
$$

Hence

$R(a, b)=p_{3}(c) p_{3}\left(c^{\perp}\right)\left[f_{2,1}(a \mid c)-f_{2,1}\left(a \mid c^{\perp}\right)\right]\left[f_{2,1}(b \mid c)-f_{2,1}\left(b \mid c^{\perp}\right)\right]$,

but

$p_{3}(c), p_{3}\left(c^{\perp}\right), f_{2,1}(a \mid c)-f_{2,1}\left(a \mid c^{\perp}\right), f_{2,1}(b \mid c)-f_{2,1}\left(b \mid c^{\perp}\right)>0$,

respectively. Therefore, $R(a, b)>0$. (Q.E.D.)

\section{Conclusions}

Let $\ell$ be a quantum logic, $p \in W_{3}, f_{2,1}$ be a conditional s-map and let $a, b, c \in L$. In fact, we can see that $c$ is a common cause to $(a, b)$, but $c$ is not necessary in order to be a common cause to $(b, a)$. We 


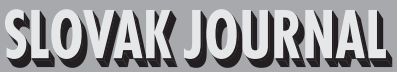 \\ 0)

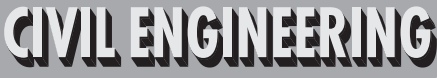

2010/4 PAGES $24-29$

notice that the whole structure of a common cause in quantum logic depends on the notion of a conditional s-map generated by an s-map $p$. In other words, the definition of a common cause will fail without an s-map.

\section{Acknowledgment}

The research presented in this paper was supported by the Slovak grant agency under the VEGA Grant No 1/0373/08 and APVV 0375-06.

\section{REFERENCES}

[1] Dvurečenskij, A., Pulmannová, S. (2000): New trends in quantum structures, Kluwer/Dordt \& Ister/Bratislava.

[2] Greechie, R. (1971): Orthogonal lattices admitting no states, J. Combin. Theory Ser. A10, 119 - 132.

[3] Garola, C. Sozzo, S.(2009): The ESR model: A proposal for a noncontextual and local Hilbert space extension of QM,EPL., ISSN, 86 2, 0295 - 5075.

[4] Khrennikov, A. (2009): Contextual Aproach to Quantum Formaliism, Springer.

[5] Khrennikov, A., Nánásiová, O.(2006): Representation theorem for observables on a quantum system, Int. Journ. of Theor. Phys, 45, $481-494$.

[6] Kolmogorov, A. N. (1956): Foundations of the theory of probability, New York: Chelsea.

[7] Nánásiová, O.(2004): Principle Conditioning, Int. Jour. of Theo. Phys., 43, 1757-1767(11).

[8] Nánásiová, O.(2003): Map for simultaneous measurements for a quantum logic, Int. Journ. of Theor. Phys., 42, 1889 $-1903$.

[9] Nánásiová, O., Khrennikov, A. (2005): Compatibility and Marginality, Int. Jour. of Theor. Phys., 46, 1083 - 1095.
[10] Nánásiová, O., Pulmannova, S. (2009): S-map and tracial states, Info. Sci., $179: 5,515$ - 520.

[11] Navara, M. (1994): An orthomodular lattice admitting no group-valued measure, Proc. of the Amer. Math. Soc., 122, 7 -12 .

[12] Pták, P., Pulmannová, S. (1991): Quantum logics, Kluwer Acad. Press, Bratislava.

[13] Reichenbach, H. (1956): The direction of time, University of California Press, Berkeley, 2, 3, 5.

[14] Rédei, M. (1997): Reichenbach's common cause principle and quantum field theory, Int. Jour. of Theo. Phys., 27, 1309 $-1321$.

[15] Rédei, M., Summers, S.J. (2002): Local primitive causality and the common cause principle in quantum field theory, Int. Jour. of Theo. Phys, 32, 335 -- 355.

[16] Renyi, A. (1947): On conditional probabilities spaces generated by a dimensionally ordered set of measures., Teorija verojatnostej i jejo primene nija, 1, 930 -- 948.

[17] Varadarajan, V.(1968): Geometry of quantum theory, Princeton, N. J., D. Van Nostrand, 1, 193. 